\title{
Constraining the cosmic ray spectrum in the vicinity of the supernova remnant W28: from sub-GeV to multi-TeV energies
}

\author{
V. H. M. Phan ${ }^{1}$, S. Gabici ${ }^{1}$, G. Morlino ${ }^{2}$, R. Terrier ${ }^{1}$, J. Vink ${ }^{3}$, J. Krause ${ }^{1}$, and M. Menu ${ }^{4}$ \\ 1 APC, Université Paris Diderot, CNRS/IN2P3, CEA/Irfu, Observatoire de Paris, Sorbonne Paris Cité, France \\ e-mail: vohongminh.phan@apc.in2p3.fr \\ 2 INAF/Osservatorio Astrofisico di Arcetri, L.go E. Fermi 5, 50125 Firenze, Italy \\ 3 GRAPPA \& Anton Pannekoek Institute for Astronomy, University of Amsterdam, Science Park 904, 1098 XH Amsterdam, \\ The Netherlands \\ ${ }^{4}$ Laboratoire de Physique des Plasmas, École Polytechnique, Univ. Paris-Sud, Université Paris-Saclay, \\ 91128 Palaiseau Cedex, France
}

Received 15 October 2019 / Accepted 28 January 2020

\begin{abstract}
Context. Supernova remnants interacting with molecular clouds are ideal laboratories to study the acceleration of particles at shock waves and their transport and interactions in the surrounding interstellar medium.

Aims. Here, we focus on the supernova remnant W28, which over the years has been observed in all energy domains from radio waves to very-high-energy gamma rays. The bright gamma-ray emission detected from molecular clouds located in its vicinity revealed the presence of accelerated $\mathrm{GeV}$ and $\mathrm{TeV}$ particles in the region. An enhanced ionization rate has also been measured by means of millimeter observations, but such observations alone cannot tell us whether the enhancement is due to low-energy (MeV) cosmic rays (either protons or electrons) or the X-ray photons emitted by the shocked gas. The goal of this study is to determine the origin of the enhanced ionization rate and to infer from multiwavelength observations the spectrum of cosmic rays accelerated at the supernova remnant shock in an unprecedented range spanning from $\mathrm{MeV}$ to multi-TeV particle energies.

Methods. We developed a model to describe the transport of X-ray photons into the molecular cloud, and we fitted the radio, millimeter, and gamma-ray data to derive the spectrum of the radiating particles.

Results. The contribution from X-ray photons to the enhanced ionization rate is negligible, and therefore the ionization must be due to cosmic rays. Even though we cannot exclude a contribution to the ionization rate coming from cosmic-ray electrons, we show that a scenario where cosmic-ray protons explain both the gamma-ray flux and the enhanced ionization rate provides the most natural fit to multiwavelength data. This strongly suggests that the intensity of CR protons is enhanced in the region for particle energies in a very broad range covering almost six orders of magnitude: from $\lesssim 100 \mathrm{MeV}$ up to several tens of $\mathrm{TeV}$.
\end{abstract}

Key words. astroparticle physics - ISM: supernova remnants - ISM: individual objects: W28 - cosmic rays - ISM: clouds

\section{Introduction}

Supernova remnants (SNRs) interacting with molecular clouds (MCs) are ideal laboratories to study the acceleration of particles at astrophysical shocks (Gabici \& Montmerle 2015). The study of such systems is of particular importance in connection with the problem of the origin of Galactic cosmic rays (CRs). This is because CRs are believed to be accelerated at SNR shocks, and injected into the interstellar medium with an energy spectrum which is a power law in momentum over a very broad range of particle energies (see e.g., Drury 2017; Gabici et al. 2019).

The SNR W28 is a middle-aged remnant (estimated age equal to few times $10^{4} \mathrm{yr}$ ) located at a distance of about $2 \mathrm{kpc}$ (Velázquez et al. 2002). It is classified as a mixed-morphology SNR with center-filled thermal X-ray emission and shell-like radio morphology (Rho \& Borkowski 2002; Dubner et al. 2000). Also, observations in $\mathrm{CO}(1-0)$ have revealed molecular gas within the field of W28 (Dame et al. 2001), concentrated in a number of massive MCs (Aharonian et al. 2008). Most importantly the detection of $1750 \mathrm{OH}$ maser from the MC located on the northeastern side of the SNR suggests that this cloud is interacting with the blast wave of the remnant (Claussen et al. 1997). The W28
SNR/MC system has been observed at all wavelengths, including radio (see Dubner et al. 2000, and references therein), millimeter (Vaupré et al. 2014), X-rays (see Zhou et al. 2014, and references therein), and high-energy and very-high-energy gamma rays (Aharonian et al. 2008; Abdo et al. 2010). This makes it an ideal target for studies of CR acceleration and escape from SNRs (e.g., Nava \& Gabici 2013, and references therein).

The MCs in the vicinity of W28 are prominent gamma-ray sources (Aharonian et al. 2008; Abdo et al. 2010). The origin of this emission is due to interactions of $\mathrm{GeV}$ and $\mathrm{TeV}$ CR protons that were accelerated in the past at the SNR shock and that now fill a vast region surrounding the remnant (e.g., Gabici et al. 2010). Remarkably, measurements performed in the millimeter domain revealed an enhanced ionization rate from the northeastern MC (Vaupré et al. 2014) which is much higher than the typical values for dense isolated MCs (Caselli et al. 1998). The ionization of the MC could be due either to the interactions of CRs (either protons or electrons) in the molecular gas, or to the presence of X-rays coming from the SNR shock-heated gas. $\mathrm{X}$-rays have been proposed as a possible source of ionization in the vicinity of a number of SNRs (Schuppan et al. 2014). 
As we see in the following, X-rays are in this case not a viable explanation for the enhanced ionization, and therefore CRs are left as the only possible ionizing agents present inside the cloud. This fact opens the possibility to combine high- and low-energy observations of the SNR/MC system (gamma rays and millimeter waves, respectively), and constrain the spectrum of CRs present in the region over an interval of particle energies of unprecedented breadth: from the $\mathrm{MeV}$ to the $\mathrm{TeV}$ domain. We show that data are best explained if an enhanced flux of CR protons is present, and if such protons are characterized by energies spanning from $\lesssim 100 \mathrm{MeV}$ up to tens of $\mathrm{TeV}$.

The paper is organized as follows: in Sect. 2 we summarize the multi-wavelength observations of the W28 SNR/MC system, in Sect. 3 we compute the photoionization rate induced in the MC by X-ray photons. The role of CRs in ionizing the gas is investigated in Sect. 4, where constraints on the CR proton and electron spectra are also obtained. We discuss our results and conclude in Sect. 5.

\section{Multi-wavelength observations of the W28 region}

In this section we review the status of the multi-wavelength observations of the W28 and its surroundings. The purple (dotdot-dashed) circle in Fig. 1 indicates the approximate contours of the SNR shell as traced by its radio emission (Dubner et al. 2000; Brogan et al. 2006). Observations in the CO molecular line revealed the presence of a number of dense $\left(\approx 10^{3} \mathrm{~cm}^{-3}\right)$ and massive $\left(\approx 10^{5} M_{\odot}\right)$ MCs in the region (Matsunaga et al. 2001; Aharonian et al. 2008). Remarkably, the H.E.S.S. collaboration reported the detection of very-high-energy gamma-ray emission from the vicinity of W28, which correlates spatially very well with the position of the MCs (Aharonian et al. 2008). The blue contours in Fig. 1 show the $4 \sigma$ significance excess in $\mathrm{TeV}$ gamma rays. The spatial correlation points towards a hadronic origin of the gamma-ray emission, which results from the interactions of CR nuclei with the dense gas that forms the MCs. Gamma-ray data are best explained by assuming that CR protons were accelerated in the past at the SNR when the shock speed was larger than the present one. Such particles then escaped the system, and now fill a large volume which encompasses all the gamma-ray-bright MCs (Fujita et al. 2009; Gabici et al. 2010; Li \& Chen 2010; Ohira et al. 2011; Nava \& Gabici 2013).

The detection of $\mathrm{OH}$ maser emission from the northeastern MC indicates that the SNR shock is currently interacting with that cloud (Claussen et al. 1997; Hewitt et al. 2008). The other TeV-bright MCs are located in the south, outside of the SNR radio boundary and therefore have not yet been reached by the shock. In the following, we focus mainly on the interaction region, and for this reason we also show, as a dashed red circle, the position and extension of the Fermi-LAT source associated to the northeastern cloud (Abdo et al. 2010; Cui et al. 2018).

The presence of the gamma-ray emission from the MCs reveals an overdensity of CRs with respect to the Galactic background, both in the $\mathrm{GeV}$ and $\mathrm{TeV}$ energy domain. In addition to that, observations of millimeter lines performed with the IRAM $30 \mathrm{~m}$ telescope (orange triangles in the figure) show that an excess in the gas ionization rate is also present at the position of the SNR/MC interaction, but not at the position of the southern MC complex (Vaupré et al. 2014; Gabici \& Montmerle 2015). Such enhanced ionization rate could be interpreted as an excess of CRs (either protons or electrons) of low energy ( $\approx \mathrm{MeV}$ energy domain). However, the SNR is a powerful thermal X-ray source (Rho \& Borkowski 2002; Zhou et al. 2014), and the X-ray photons might also penetrate the cloud and be responsible for the

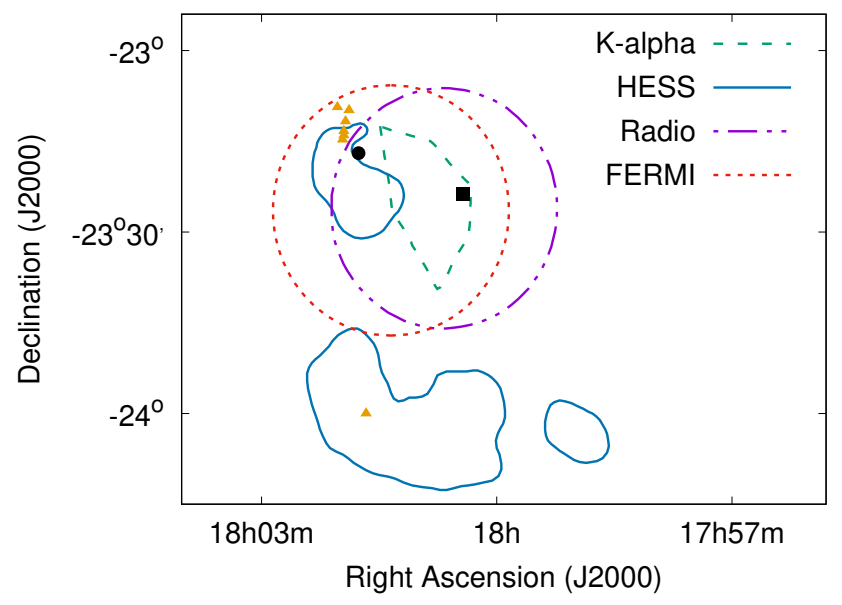

Fig. 1. Contour map for the W28 region. The approximate radio boundary of the SNR shell is shown as a purple dot-dot-dashed circle. The solid blue contours represent the $4 \sigma$ significance excess $\mathrm{TeV}$ emission observed by H.E.S.S. (Aharonian et al. 2008). The short-dashed red circle is the best-fit disk size for the Fermi-LAT GeV source associated to the northeastern MC (Cui et al. 2018). The enhanced region of $\mathrm{Fe} \mathrm{I} \mathrm{K} \alpha$ line emission is the area enclosed by the dashed green line (Nobukawa et al. 2018). The CR ionization rate has been measured from IRAM observations pointed in the directions indicated by the yellow triangles (Vaupré et al. 2014). The filled black circle and square indicate the centroids of the X-ray emission for the northeast and central X-ray sources, respectively (Rho \& Borkowski 2002).

enhanced ionization rate, as was proposed for other SNR/MC systems (Schuppan et al. 2014). The spatial morphology of the $\mathrm{X}$-ray emission is quite extended, and can be roughly described as the sum of two extended sources, whose centroids are shown in Fig. 1 as a filled black square (central source, C) and circle (northeastern source, NE). Determining whether the enhanced ionization rate is due to $\mathrm{CR}$ protons, electrons, or X-ray photons is one of the goals of this paper (see Sect. 3 ).

Finally, additional constraints on the origin of the enhanced ionization rate can be obtained from hard X-ray observations of W28 performed by Suzaku (Nobukawa et al. 2018). These observations revealed the presence of the Fe I K $\alpha$ line in the $\mathrm{X}$-ray spectrum. This line is produced by interactions between low-energy (MeV domain) CRs and cold gas, and it is therefore tempting to propose a common origin for the line emission and the excess in the ionization rate measured in the northeastern MC. Puzzlingly, Fe I K $\alpha$ line emission has been detected from a region (green dashed contour in Fig. 2) close to but not coincident with the position of the gamma-ray bright northeastern MC.

\section{Photoionization}

Based on XMM-Newton observations, Zhou et al. (2014) claimed that the X-ray emission from the SNR W28 is predominantly thermal, with a possible subdominant nonthermal contribution from source NE, and an indication for the presence of a multi-temperature gas for source C (see also Rho \& Borkowski 2002).

In order to estimate the level of photoionization induced by the SNR X-ray emission in the northeastern MC, we make use of the spectral fits to XMM data obtained by Zhou et al. (2014). For simplicity, we consider single-temperature nonequilibrium ionization models where the abundances, ionization timescale, and plasma temperature are allowed to vary to fit the X-ray emission 
of both the NE and $\mathrm{C}$ source (vnei models in $\mathrm{XSPEC}^{1}$ ). We refer the interested reader to Vink (2012) (see also Borkowski et al. 2001) for a more detailed discussion of these plasma models. The best-fit parameters are provided in Table 1, where the abundances are defined as the ratio between the abundances of the sources and the abundances in the Solar System. Even though more sophisticated models obtained by adding a power law or a hot component with higher temperature to the spectrum of the $\mathrm{NE}$ and $\mathrm{C}$ sources could improve the spectral fits (see some of the proposed models by Zhou et al. 2014), this has very little effect in our estimate of the photoionization rate.

The intensity of the X-ray radiation inside the northeastern $\mathrm{MC}$ has been computed by assuming that the X-ray sources NE and $\mathrm{C}$ are point-like and located at the position of the two centroids of the X-ray emission (filled black circle and square in Fig. 1). The contribution to the density of X-ray photons at a given location $\boldsymbol{r}$ away from the source can be computed as:

$n_{\mathrm{ph}}(E, \boldsymbol{r})=\frac{F(E) D_{\mathrm{s}}^{2}}{c r^{2}} \exp \left[-\frac{n_{\mathrm{H}_{2}} \sigma_{\mathrm{abs}}(E)}{f_{\mathrm{H}_{2}}} \mathrm{~d}(r)\right]$,

where $F(E)$ is the X-ray source's unabsorbed differential photon flux, $D_{\mathrm{s}} \sim 2 \mathrm{kpc}$ is the distance from the source to Earth, $c$ is the speed of light, $n_{\mathrm{H}_{2}}$ is the density of $\mathrm{H}_{2}$ molecules in the absorbing medium (which in this case is the MC), $f_{\mathrm{H}_{2}}=n_{\mathrm{H}_{2}} /\left(2 n_{\mathrm{H}_{2}}+n_{\mathrm{H}}\right)$ is the fractional density of $\mathrm{H}_{2}$ molecules relative to the total number of $\mathrm{H}$ atom, $\sigma_{\mathrm{abs}}(E)$ is the photoelectric absorption crosssection per $\mathrm{H}$ atom, and $\mathrm{d}(r)$ is the distance travelled inside of the cloud by the X-ray photons that reached a distance $r$ from the source (see Fig. 2). For MCs, it is appropriate to set $f_{\mathrm{H}_{2}} \sim 0.5$ (see e.g., Vaupré et al. 2014), and we further assume solar abundances to describe the gas in the MC. This latter assumption allows us to use the absorption cross-section $\sigma_{\text {abs }}(E)$ taken from Morrison \& McCammon (1983). In fact, the exact value adopted for the element abundances in the MC might have some impact: changing from the solar abundances to the ones of the NE source as reported in Table $1(\sim 0.3$ times the solar abundances for most elements) would increase the estimate of the photoionization rate by a factor of approximately three (see Fig. 3).

With Eq. (1) at hand, the photoionization rate $\xi_{\mathrm{ph}}$ induced by X-ray photons inside the cloud can be obtained following the approach presented in Maloney et al. (1996). Due to the relatively low temperatures of the emitting plasmas $(<1 \mathrm{keV})$, the contribution from Compton scattering to the photoionization rate can be safely neglected. Moreover, most of the ionization in the $\mathrm{MC}$ will be induced by secondary electrons generated as a result of the X-ray photoionization, meaning that we can write:

$\xi_{\mathrm{ph}}(\boldsymbol{r})=2 f_{\mathrm{H}_{2}} \sum_{\mathrm{s}} \int_{I\left(\mathrm{H}_{2}\right)}^{E_{\max }} \sigma_{\mathrm{abs}}(E) c n_{\mathrm{ph}}^{\mathrm{s}}(E, \boldsymbol{r}) M_{\mathrm{sec}}(E) \mathrm{d} E$,

where the sum indicates that both X-ray sources ( $s=\mathrm{NE}$ and $\mathrm{C}$ ) are considered. Here, $I\left(\mathrm{H}_{2}\right) \approx 15.6 \mathrm{eV}$ is the ionization potential of $\mathrm{H}_{2}$, and $M_{\mathrm{sec}}(E)=\left[E-I\left(\mathrm{H}_{2}\right)\right] / W$ is the mean multiplicity for ionization by a secondary electron in a $\mathrm{H}_{2}$ gas, with $W \sim 40 \mathrm{eV}$ (see Dalgarno et al. 1999; Dogiel et al. 2013).

Before proceeding, a discussion of the geometry of the problem is in order (see Fig. 2). We model the X-ray emission from the SNR as two point sources located at the position of the centroids of the emission (Rho \& Borkowski 2002). The ionization rate in the northeastern $\mathrm{MC}$ has been measured along several lines of sight (yellow triangles in Fig. 1, Vaupré et al. 2014). To maximise the effect of photoionization, we have chosen to

\footnotetext{
1 https://heasarc.gsfc . nasa.gov/xanadu/xspec/
}

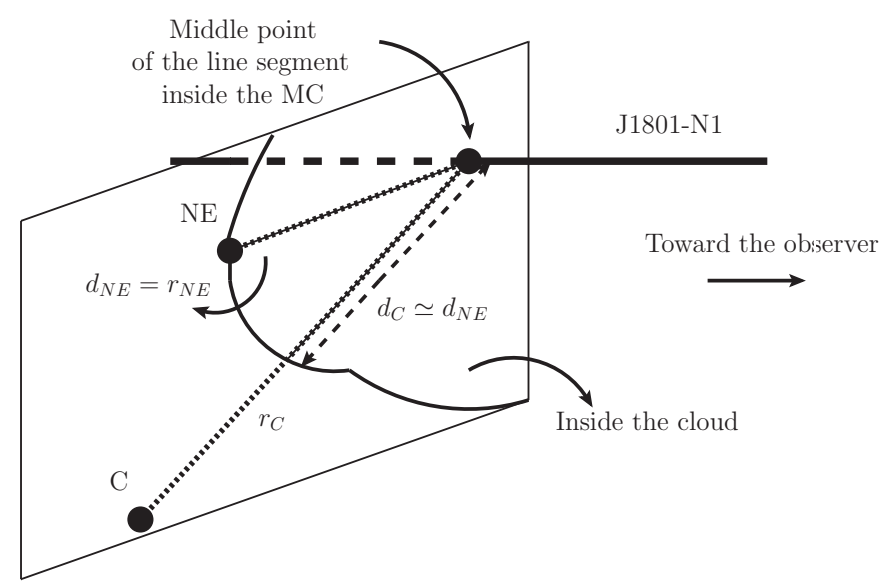

Fig. 2. Adopted geometry for the calculation of photoionization. The centroids of the X-ray emission are indicated by the points labeled NE and $\mathrm{C}$, and the line of sight labeled J1801-N1 is the IRAM pointing closest to the X-ray sources.

Table 1. Fit parameters of the vnei model for the X-ray sources adopted from Zhou et al. (2014).

\begin{tabular}{lcc}
\hline \hline Objects & NE & $\mathrm{C}$ \\
\hline$k T_{\mathrm{c}}(\mathrm{keV})$ & 0.33 & 0.60 \\
$\tau_{\mathrm{c}}\left(10^{11} \mathrm{~cm}^{-3} \mathrm{~s}\right)$ & 6.00 & 2.35 \\
$\mathrm{Abun}{ }^{(1),(2)}$ & 0.26 & 0.12 \\
$\mathrm{Si}$ & 0.40 & 0.16 \\
$\mathrm{~S}$ & 0.88 & 0.39 \\
$\mathrm{Fe}$ & 0.22 & 0.10 \\
$\phi_{\mathrm{s}}{ }^{(3)}\left(10^{-11} \mathrm{erg} \mathrm{cm}^{-2} \mathrm{~s}^{-1}\right)$ & 6.61 & 10.43 \\
\hline
\end{tabular}

Notes. ${ }^{(1)}$ All the abundances are relative to solar ones (see Sect. 3 for more explanations). ${ }^{(2)}$ Abundances of $\mathrm{C}, \mathrm{N}, \mathrm{O}, \mathrm{Ne}, \mathrm{Mg}, \mathrm{Ar}, \mathrm{Ca}$, and Ni. ${ }^{(3)}$ Unabsorbed fluxes in the $0.3-5.0 \mathrm{keV}$ band.

Table 2. Coordinates and relative distances of the two X-ray sources and of the line of sight J1801-N1.

\begin{tabular}{lcccc}
\hline \hline Objects & $\begin{array}{c}\alpha \\
(\mathrm{hms})\end{array}$ & $\begin{array}{c}\delta \\
\left({ }^{\circ}{ }^{\prime \prime}{ }^{\prime \prime}\right)\end{array}$ & $\begin{array}{c}d_{\mathrm{NE}} \\
(\mathrm{pc})\end{array}$ & $\begin{array}{c}d_{\mathrm{C}} \\
(\mathrm{pc})\end{array}$ \\
\hline NE source & 180145.7 & -231658.3 & - & - \\
C source & 180025.5 & -232347.2 & - & - \\
J1801-N1 & 180158.0 & -231444.0 & 2.10 & 13.43 \\
\hline
\end{tabular}

study the line of sight which is the closest to the centroids of the X-ray emission, which has been labeled by Vaupré et al. (2014) as J1801-N1. This line of sight is also the one characterized by the smaller measured value of the density of $\mathrm{H}_{2}$, which is equal to $n_{\mathrm{H}_{2}} \sim 600 \mathrm{~cm}^{-3}$ (Vaupré et al. 2014). In the following, we assume that this density characterizes the entire cloud. This is of course not true, given that larger values of this quantity have been estimated by Vaupré et al. (2014) for all the lines of sight other than J1801-N1, but such an assumption will provide us with the most optimistic (larger) estimate of the photoionization rate in the cloud. We further assume that the two X-ray sources lay on a plane orthogonal to the line of sight J1801-N1, and that the northeastern MC is spatially symmetric with respect to that plane. The coordinates of the two X-ray sources and of the line of sight J1801-N1 are listed in Table 2 together with their relative distances. 

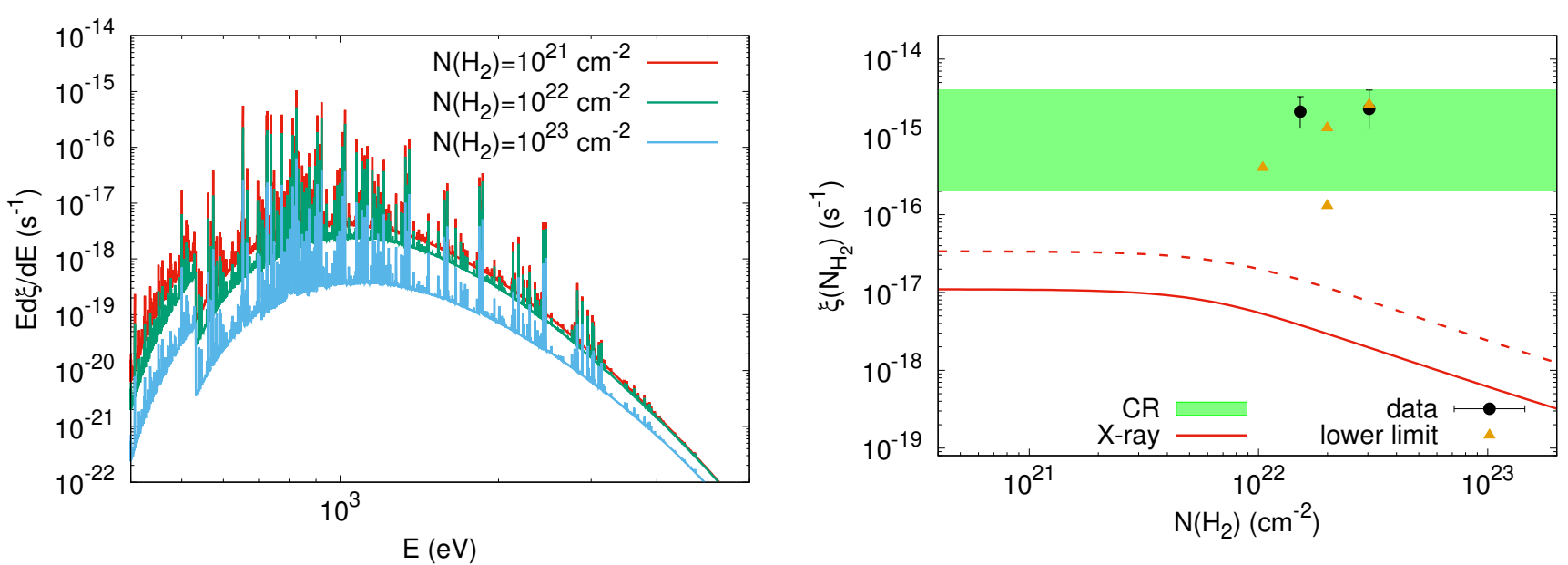

Fig. 3. Left: average differential ionization rate for different assumed gas column densities along the line of sight N1 and solar abundance. Right: predictions for the photoionization rate are shown by the solid and dashed red lines (for the cloud with solar abundances and the abundances of the NE source, respectively). Observational data taken from Vaupré et al. (2014) are presented as filled circles (measurements) and filled triangles (lower limits). The shaded region indicates a reference range of values of the ionization rate which has been used to constrain the CR spectrum (see text).

Adopting the geometry presented in Fig. 2, we can now estimate the X-ray photo-ionization rate as a function of the gas column density along the line of sight $N\left(\mathrm{H}_{2}\right)$. Figure 3 shows the differential photo-ionization rate averaged along the line of sight for different values of the column densities along the line of sight: $N\left(\mathrm{H}_{2}\right)=10^{21}, 10^{22}, 10^{23} \mathrm{~cm}^{-2}$, respectively. For typical values in the range $N\left(\mathrm{H}_{2}\right) \approx 10^{21}-10^{23} \mathrm{~cm}^{-2}$, the left panel of Fig. 3 shows that the contribution to ionization comes mainly from X-ray photons with energy in the range from $\lesssim 1 \mathrm{keV}$ to a few $\mathrm{keV}$.

The right panel of Fig. 3 shows the expected photo-ionization rates as a function of the gas column density for a cloud of solar (solid red curve) and 0.3 times solar metallicity (dashed red curve). These predictions can be compared with the measurements of the ionization rate in the northeastern cloud (data points and lower limits in the figure). One can clearly see that the contribution from X-rays to the observed ionization rate is negligible even in the most optimistic scenario considered here. Therefore, the ionization rate must be due to CRs, either protons (or nuclei) or electrons. We consider this scenario in the following section.

\section{Cosmic-ray induced ionization}

The gamma-ray emission detected from the MCs in the vicinity of the SNR W28 is interpreted as the result of hadronic interactions between CR nuclei (mostly protons) accelerated in the past at the SNR shock. These particles escaped the remnant and now fill a vast region that encompasses the clouds. The gammaray emission results from the decay of neutral pions produced in inelastic proton-proton interactions. The energy threshold to produce pions at rest is $T_{\mathrm{p}}^{\text {th }} \sim 280 \mathrm{MeV}$. Therefore, gamma-ray observations can be used, generally, to determine the shape of the spectrum of CR protons of kinetic energy exceeding $T_{\mathrm{p}}^{\text {th }}$ contained in the MCs.

It is definitely less straightforward to infer the energy spectrum of the CR electrons contained within the cloud. The SNR W28 is a bright synchrotron radio source (Dubner et al. 2000), and this indicates the presence of relativistic electrons and magnetic field in the region. Even though the morphology of the radio emission does not correlate with that of molecular clouds, the brightest region in radio roughly coincides with the position of the northeastern cloud.

In the following, we investigate the possibility that either CR protons or electrons are the responsible for the enhanced ionization rate measured from the northeastern cloud. The $\mathrm{H}_{2}$ ionization rate induced by proton and electron CRs could be obtained as in Padovani et al. (2009) (see also Phan et al. 2018; Recchia et al. 2019):

$$
\begin{aligned}
\zeta_{\mathrm{p}}\left(\mathrm{H}_{2}\right)= & \int_{I}^{E_{\max }} f_{a}(E) v_{\mathrm{p}}\left[1+\phi_{\mathrm{p}}(E)\right] \sigma_{\mathrm{ion}}^{\mathrm{p}}(E) \mathrm{d} E \\
& +\int_{0}^{E_{\max }} f_{a}(E) v_{\mathrm{p}} \sigma_{\mathrm{ec}}(E) \mathrm{d} E \\
\zeta_{\mathrm{e}}\left(\mathrm{H}_{2}\right)= & \int_{I}^{E_{\max }} f_{a}(E) v_{\mathrm{e}}\left[1+\phi_{\mathrm{e}}(E)\right] \sigma_{\mathrm{ion}}^{\mathrm{e}}(E) \mathrm{d} E,
\end{aligned}
$$

where $\sigma_{\text {ion }}^{\mathrm{p}}, \sigma_{\mathrm{ec}}$, and $\sigma_{\text {ion }}^{\mathrm{e}}$ are the proton ionization cross-section, the electron capture cross-section, and the electron ionization cross-section, respectively (see Padovani et al. 2009; Krause et al. 2015, and references therein). It should be noted that, following Krause et al. (2015), the two ionization cross-sections are included in the computation with fully relativistic corrections. Here, $I=15.603 \mathrm{eV}$ is the ionization potential of $\mathrm{H}_{2}$ while $\phi_{\mathrm{p}}(E)$ and $\phi_{\mathrm{e}}(E)$ are the average secondary ionization per primary ionization computed as in Krause et al. (2015). In general, the values of $\phi_{\mathrm{p}}(E)$ and $\phi_{\mathrm{e}}(E)$ are approximately one within the energy range that is relevant for ionization and thus secondary electrons would contribute around $50 \%$ of the CR-induced ionization rate.

\subsection{Cosmic-ray protons and nuclei}

Let us assume that the spectrum of CR protons in the cloud can be described as a power law in momentum $\propto p^{-\left(\delta_{\mathrm{p}}+2\right)}$ as expected if protons are accelerated at the SNR shock via a first-order Fermi mechanism. In terms of the particle kinetic energy $T_{\mathrm{p}}$ this writes:

$n_{\mathrm{p}}\left(T_{\mathrm{p}}\right)=A_{\mathrm{p}}\left(T_{\mathrm{p}}+m_{\mathrm{p}} c^{2}\right)\left[T_{\mathrm{p}}^{2}+2 T_{\mathrm{p}} m_{\mathrm{p}} c^{2}\right]^{-\frac{\delta_{\mathrm{p}}+1}{2}}$, 
where all energies are in $\mathrm{GeV}$ and $A_{\mathrm{p}}$ is a normalization factor. Both $A_{\mathrm{p}}$ and the spectral index $\delta_{\mathrm{p}}$ can be obtained by fitting the gamma-ray data.

Assuming that the observed gamma rays are produced by proton-proton interactions, the expected gamma-ray flux measured at Earth would be:

$$
\begin{aligned}
\phi\left(E_{\gamma}\right)= & \frac{M_{\mathrm{cl}}}{4 \pi D_{\mathrm{s}}^{2} m_{\mathrm{avg}}} \int_{T_{\mathrm{p}}^{\min }}^{T_{\mathrm{p}}^{\max }} 4 \pi J_{\mathrm{p}}\left(T_{\mathrm{p}}\right) \\
& \times \varepsilon\left(T_{\mathrm{p}}\right) \frac{\mathrm{d} \sigma_{\mathrm{pp}}\left(T_{\mathrm{p}}, E_{\gamma}\right)}{\mathrm{d} E_{\gamma}} \mathrm{d} T_{\mathrm{p}},
\end{aligned}
$$

where $J_{\mathrm{p}}\left(T_{\mathrm{p}}\right)=(v / 4 \pi) n_{\mathrm{p}}\left(T_{\mathrm{p}}\right)$ is the CR proton intensity as a function of the particle kinetic energy $T_{\mathrm{p}}(v$ is the particle velocity). Moreover, $M_{\mathrm{cl}}$ is the mass of the whole cloud $\left(\sim 5 \times 10^{4} M_{\odot}\right.$, see Aharonian et al. 2008), $m_{\text {avg }}$ is the average atomic mass of the gas from the $\mathrm{MC}$ (with solar abundance $m_{\mathrm{avg}} \simeq 1.4 m_{\mathrm{p}}$ ), $T_{\mathrm{p}}^{\max }$ is the maximum kinetic energy of the accelerated particle (its exact value is irrelevant, as long as $\gg 100 \mathrm{TeV}$ ), and $T_{\mathrm{p}}^{\mathrm{min}}$ is the threshold energy for $\pi^{0}$ production in proton-proton interactions. Also, $\mathrm{d} \sigma_{\mathrm{pp}}\left(T_{\mathrm{p}}, E_{\gamma}\right) / \mathrm{d} E_{\gamma}$ is the differential cross-section for gamma-ray production and $\varepsilon\left(T_{\mathrm{p}}\right)$ is the nuclear enhancement factor to take into account gamma-ray production from nucleusnucleus interaction (both taken from Kafexhiu et al. 2014). A fit to the gamma-ray data is shown in Fig. 4 (left panel), where data points are from Aharonian et al. (2008) and Abdo et al. (2010). The values obtained for $A_{\mathrm{p}}$ and $\delta_{\mathrm{p}}$ are reported in the first row of Table 3. Of course, the CRs responsible for the gamma ray emission are characterized by particle energies in the $\mathrm{GeV}$ and $\mathrm{TeV}$ domains, and therefore their contribution to the ionization rate is negligible (e.g., Morlino \& Gabici 2015; Padovani et al. 2009; Phan et al. 2018).

In order to estimate the possible contribution from CR protons to the measured ionization rate in the cloud, we extrapolate the power law spectrum obtained after fitting the gamma-ray data down to the $\mathrm{MeV}$ energy domain. In other words, we assume a spectrum as in Eq. (5) down to an arbitrary particle kinetic energy $T_{\mathrm{c}}$. We then compute the ionization rate due to CR protons using Eq. (3) and multiply by a factor $\eta=1.5$ to take into account the contribution from CR nuclei (Padovani et al. 2009). This allows us to constrain the value of $T_{\mathrm{c}}$ so that the ionization rate falls in the range indicated by the green shaded region in Fig. 3. The range of values $T_{\mathrm{c}}^{\min }<T_{\mathrm{c}}<T_{\mathrm{c}}^{\max }$ obtained in this way are reported in Table 3.

There are several reasons to envisage a change in the CR proton spectrum at certain particle energies $T_{\mathrm{c}}$. Let us first consider the scenario where the region of enhanced ionization is upstream of the shock and the ionizing CRs have already escaped the remnant. In this case, the range of possible numerical values of $T_{\mathrm{c}}$ can be estimated as follows.

1. Cosmic-ray protons have been produced at the SNR shock $\tau_{\text {inj }}$ years ago, and since then they have suffered energy losses (mainly ionization losses) in the dense gas, over a characteristic time $\tau_{\text {ion }}\left(T_{\mathrm{p}}\right)$, which is proportional to the gas density and an increasing function of particle energy (see Fig. 2 in Phan et al. 2018). In fact, energy losses can be effective only for particles characterized by an energy smaller than $T_{\mathrm{c}}$, defined as $\tau_{\text {ion }}\left(T_{\mathrm{c}}\right)=$ $\tau_{\text {inj }}$, because particles of higher energy simply do not have time to cool. The maximum possible value for $\tau_{\text {inj }}$ is of course the age of the SNR $\tau_{\text {age }} \approx 4 \times 10^{4} \mathrm{yr}$ (Gabici et al. 2010), which provides an upper limit for $T_{\mathrm{c}}$. For a typical gas density of $n_{\mathrm{H}_{2}} \sim 10^{3} \mathrm{~cm}^{-3}$ this gives $T_{\mathrm{c}} \lesssim 4 \times 10^{2} \mathrm{MeV}$, which is quite close to the value of $T_{\mathrm{c}}^{\max }$ in Table 3.
2. Cosmic-ray protons have to penetrate deep into the cloud in order to ionize the gas there. If we use $\tau_{\mathrm{p}}$ to refer to the time it takes them to reach the center of the cloud moving a distance $L$ away from the position of the shock, we can estimate $T_{\mathrm{c}}$ by imposing $\tau_{\text {ion }}=\tau_{\mathrm{p}}$. The shortest possible penetration time $\tau_{\mathrm{p}}$ is obtained after assuming that CR protons move along straight lines at a velocity $v \sim \sqrt{2 T_{\mathrm{c}} / m_{\mathrm{p}}}$. If we assume that $\mathrm{CR}$ protons have to cross a gas column density of $\sim 3 \times 10^{22}\left(n_{\mathrm{H}_{2}} / 10^{3} \mathrm{~cm}^{-3}\right)(L / 10 \mathrm{pc}) \mathrm{cm}^{-2}$ we get $T_{\mathrm{c}} \gtrsim 7 \mathrm{MeV}$, which is a factor of a few smaller than $T_{\mathrm{c}}^{\mathrm{min}}$ in Table 3.

It should be noted that, in this scenario, the range of possible values for $T_{\mathrm{c}}$ obtained by means of the phenomenological consideration made above overlaps very well with the range of values obtained observationally (Table 3 ), that is, by fitting the millimeter and gamma-ray data simultaneously.

Another possible scenario is that the SNR shock has overrun the region of enhanced ionization, engulfing it with low-energy CRs which are still inside the shell in the downstream region. This is similar to the case of the region of enhanced ionization W51C-E which has been shown to be in the downstream region of the SNR W51C (see Dumas et al. 2014 for more detailed discussion). If this is true then we may expect a hardening in the low-energy part of the spectrum below $T_{\mathrm{c}}$ due to the difference in spectral features of the escaped CRs at high energy and the still confined CRs at low energy. However, our understanding of the escape of CRs from SNR shocks is still quite poor, making an accurate estimate of the numerical value of $T_{\mathrm{c}}$ problematic.

Independently of the scenario, the important point that needs to be stressed is that gamma-ray observations allow us to constrain the spectrum of CR protons for particle energies above $\approx 1 \mathrm{GeV}$. Therefore, the explanation of the enhanced ionization rate requires extrapolation of the proton spectrum only by a factor of $\approx 3-30$ down to lower particle energies.

It follows that the presence of an excess of CR protons characterized by a relatively steep power-law spectrum $\left(\delta_{\mathrm{p}} \approx 2.8\right)$ extending from the $\mathrm{MeV}$ to multi-TeV domain can explain very naturally both the bright gamma-ray emission from the northeastern cloud and the observed enhancement in the ionization rate.

\subsection{Cosmic-ray electrons}

As stated above, the decay of neutral pions produced in inelastic interactions between CR protons (and nuclei) and the dense gas in the cloud provides the most natural explanation for the gamma-ray emission observed in the $\mathrm{GeV}$ and $\mathrm{TeV}$ energy domain (see e.g., Aharonian et al. 2008; Nava \& Gabici 2013). Leptonic models where the emission is due to nonthermal Bremsstrahlung have been shown to be problematic, as they require the SNR to accelerate the same number of nuclei and electrons, and only provide a good fit to gamma-ray data if unrealistic values of the local magnetic field strength and gas density are assumed (Abdo et al. 2010).

This implies that the nonthermal Bremsstrahlung emission from CR electrons must provide a subdominant contribution to the observed gamma-ray emission. If $n_{\mathrm{e}}\left(E_{\mathrm{e}}\right)=A_{\mathrm{e}}\left(E_{\mathrm{e}} / \mathrm{GeV}\right)^{-\delta_{\mathrm{e}}}$ is the density of relativistic electrons of energy $E_{\mathrm{e}}$ inside the cloud, the expected Bremsstrahlung emission can be roughly estimated as:

$E_{\gamma}^{2} L_{\mathrm{B}}\left(E_{\gamma} \sim E_{\mathrm{e}}\right) \sim \frac{E_{\mathrm{e}}^{2} n_{\mathrm{e}}\left(E_{\mathrm{e}}\right) V_{\mathrm{cl}}}{\tau_{\mathrm{B}}}$,

where

$\tau_{\mathrm{B}} \sim 4 \times 10^{7}\left(\frac{n_{\mathrm{H}}}{\mathrm{cm}^{-3}}\right)^{-1} \mathrm{yr}$ 

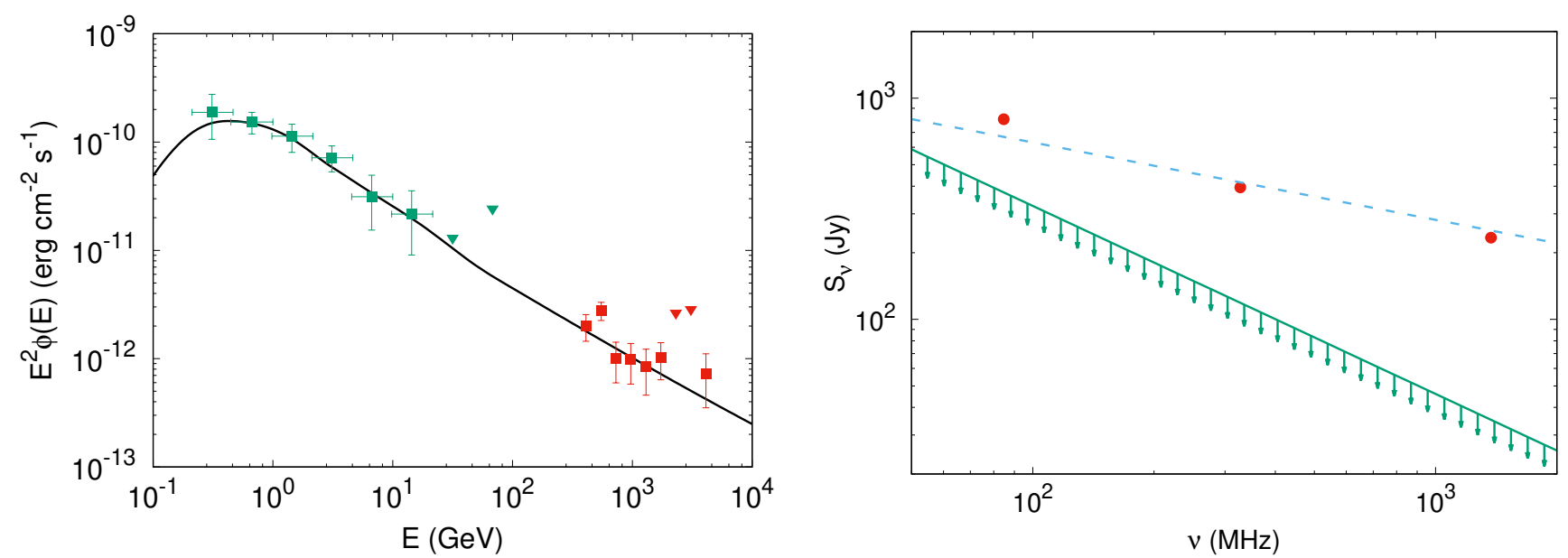

Fig. 4. Left: gamma-ray differential energy spectrum of the northeastern MC (J1801-233). Fermi-LAT and HESS data have been fitted with a hadronic model (see Aharonian et al. 2008; Abdo et al. 2010, for references). Right: synchrotron emission spectrum observed from the entire SNR (Kovalenko et al. 1994; Dubner et al. 2000). A fit to data is shown as a dashed line. The maximum contribution from the northeastern cloud is also shown (see text for details).

Table 3. Fit parameters for the CR proton spectrum and upper limit for the CR electron spectrum.

\begin{tabular}{lccc}
\hline \hline Species & $\begin{array}{c}A_{\mathrm{p}, \mathrm{e}} \\
\left(\mathrm{eV}^{-1} \mathrm{~cm}^{-3}\right)\end{array}$ & $\delta_{\mathrm{p}, \mathrm{e}}$ & $\begin{array}{c}T_{\mathrm{c}}^{\min }-T_{\mathrm{c}}^{\max } \\
(\mathrm{MeV})\end{array}$ \\
\hline Proton & $\begin{array}{c}3.15 \times 10^{-17} \\
\ll 6.4 \times 10^{-19}\end{array}$ & $\begin{array}{c}2.76 \\
2.7\end{array}$ & $\begin{array}{c}26-320 \\
\ll 20-130 \\
\text { Electron }\end{array}$ \\
\hline
\end{tabular}

is the energy loss time due to Bremsstrahlung in a gas characterized by an atomic hydrogen density $n_{\mathrm{H}}$ (Aharonian 2004) and $V_{\mathrm{cl}}$ is the volume of the cloud.

By imposing that the Bremsstrahlung flux $E_{\gamma}^{2} L_{\mathrm{B}} /\left(4 \pi D_{\mathrm{s}}^{2}\right)$ should be significantly smaller than the observed one $E_{\gamma}^{2} \phi\left(E_{\gamma}\right)$ we get:

$E_{\mathrm{e}}^{2} n_{\mathrm{e}}\left(E_{\mathrm{e}}\right) \ll 4 \pi D_{\mathrm{s}}^{2}\left(\frac{\tau_{\mathrm{B}}}{n_{\mathrm{H}}}\right) E_{\gamma}^{2} \phi\left(E_{\gamma}\right) m_{\mathrm{p}} M_{\mathrm{cl}}^{-1}$,

where we introduced the source distance $D_{\mathrm{s}}$ and the cloud mass $M_{\mathrm{cl}}=m_{\mathrm{p}} n_{\mathrm{H}} V_{\mathrm{cl}}$. At a photon energy equal to $E_{\gamma}=$ $1 \mathrm{GeV}$ the observed gamma-ray flux is roughly $E_{\gamma}^{2} \phi\left(E_{\gamma}\right) \approx$ $10^{-10} \mathrm{erg} \mathrm{cm}^{-2} \mathrm{~s}^{-1}$, and above such energy the spectrum can be described by a power law $E_{\gamma}^{-\alpha}$ with $\alpha \sim 2.7$ (Abdo et al. 2010; Aharonian et al. 2008). This gives:

$E_{\mathrm{e}}^{2} n_{\mathrm{e}}\left(E_{\mathrm{e}}\right) \ll 10^{-12}\left(\frac{E_{\mathrm{e}}}{\mathrm{GeV}}\right)^{-0.7} \mathrm{erg} \mathrm{cm}^{-3}$

which corresponds to $A_{\mathrm{e}} \ll 6.4 \times 10^{-19} \mathrm{eV}^{-1} \mathrm{~cm}^{-3}$.

We note that the ratio between the intensity of $\mathrm{CR}$ protons and electrons inside the cloud is $A_{\mathrm{p}} / A_{\mathrm{e}} \gg 50$ for particle energies of the order of $1 \mathrm{GeV}$, and varies very little for larger particle energies (because $\delta_{\mathrm{p}} \sim \delta_{\mathrm{e}}$ ).

The constraints obtained above on the electron spectrum in the cloud can be used to estimate the contribution given by such electrons to the observed radio emission from the SNR. Radio observations of W28 have been performed in a frequency range spanning from $\lesssim 100 \mathrm{MHz}$ to several gigahertz. The radio spectrum is shown in the right panel of Fig. 4: the flux at $1.4 \mathrm{GHz}$ is $S_{1.4} \sim 246 \mathrm{Jy}$, and the radio spectrum can be described by a power law $S_{v} \propto v^{-\alpha}$ with $\alpha \sim 0.35$ (Dubner et al. 2000). Even though the radio emission is observed from a region which is spatially more extended than the northern cloud, the radio brightness roughly peaks at that position. Therefore, it is natural to ask whether a contribution to the radio emission might come from relativistic electrons located inside the cloud.

Electrons of energy $E$ emit synchrotron photons of frequency (Aharonian 2004):

$v_{\mathrm{s}} \sim v_{\mathrm{c}} / 3=\frac{\Omega_{\mathrm{L}}}{2}\left(\frac{E_{\mathrm{e}}}{m_{\mathrm{e}} c^{2}}\right)^{2} \sim 0.2\left(\frac{B}{40 \mu \mathrm{G}}\right)\left(\frac{E_{\mathrm{e}}}{\mathrm{GeV}}\right)^{2} \mathrm{GHz}$,

where $v_{\mathrm{c}}$ is the critical synchrotron frequency, $\Omega_{\mathrm{L}}$ the nonrelativistic Larmor frequency, and $m_{\mathrm{e}} c^{2}$ the electron rest mass energy. This implies that the observed radio emission is produced by electrons of energies in the range $0.1 \lesssim$ $\left(E_{\mathrm{e}} / \mathrm{GeV}\right)(B / 40 \mu \mathrm{G})^{1 / 2} \lesssim 10$. Electrons of such energies emit Bremsstrahlung in the Fermi energy domain.

The value of the magnetic field in cloud $B$ has been normalized to what is expected from the observational relationship $B \approx 10\left(n / 100 \mathrm{~cm}^{-3}\right)^{0.5} \mu \mathrm{G}$ between the cloud magnetic field and the density of the gas (Crutcher 1999). For the northeastern cloud, Aharonian et al. (2008) estimated a gas density equal to $n \sim 1.4 \times 10^{3} \mathrm{~cm}^{-3}$, which would give $B \sim 37 \mu \mathrm{G}$.

The upper limit on the CR electron spectrum obtained above (Eq. (10)) can now be used to estimate the maximum contribution to the observed synchrotron emission coming from the northeastern cloud. This is shown in the right panel of Fig. 4. The contribution is subdominant at all frequencies. This implies that the radio emission is largely produced by electrons located outside the cloud.

Therefore, it is not at all straightforward to estimate the possible contribution of $\mathrm{CR}$ electrons to the ionization rate in the cloud. Even though we do not know the spectral slope $\delta_{\mathrm{e}}$ and normalization $A_{\mathrm{e}}$ of the CR electrons inside the MC, we have obtained an upper limit for the latter value from gamma-ray observations (see Table 3) which applies to electrons of particle energy above $E_{\mathrm{e}}^{\mathrm{min}} \approx E_{\gamma}^{\mathrm{min}} \approx 0.2 \mathrm{GeV}$. Electrons of energy larger than $E_{\mathrm{e}}^{\mathrm{min}}$ and characterized by an intensity well below the upper limit reported in Eq. (10) would produce an ionization rate of the order of $\ll 1.4 \times 10^{-16} \mathrm{~s}^{-1}$ (the upper limit for the electron-induced ionization rate is calculated using Eq. (4)). 
Therefore, as one can easily see from Fig. 3, electrons of energy exceeding $E_{\mathrm{e}}^{\mathrm{min}}$ cannot explain the observed ionization rate in the cloud.

An extrapolation of the upper limit electron spectrum to much lower particle energies might possibly result in a value of the ionization rate comparable with observations. Using again the upper limit for $A_{\mathrm{e}}$, together with a spectral index of $\delta_{\mathrm{e}} \simeq 2.7$, which is quite steep and therefore maximizes the impact of electrons, we find that values of $T_{\mathrm{c}}$ well below $20-130 \mathrm{MeV}$ are needed in order to match the observed ionization rate (see Table 3). However, the validity of such an extrapolation is questionable since the spectral index for electrons is not as well constrained as that of protons.

Even though we cannot reach a firm conclusion, explaining the excess in the ionization rate with $\mathrm{CR}$ protons is very natural (Sect. 4.1) and therefore the hadronic scenario remains, in our view, the preferred one.

\subsection{Constraints from the $6.4 \mathrm{keV}$ line emission}

Interestingly, the SNR W28 is also observed by Suzaku in the $\mathrm{Fe}$ I K $\alpha$ line emission and quite recently Nobukawa et al. (2018) found that there is an excess of this emission from the central region of the remnant with intensity $I_{6.4 \mathrm{keV}}^{\mathrm{en}}=(3.14 \pm$ $0.43) \times 10^{-8} \mathrm{~cm}^{-2} \mathrm{~s}^{-1}$ arcmin $^{-2}$ compared to the background of the Galactic ridge X-ray emission $I_{6.4 \mathrm{keV}}^{\mathrm{bg}}=(2.33 \pm 0.29) \times$ $10^{-8} \mathrm{~cm}^{-2} \mathrm{~s}^{-1} \operatorname{arcmin}^{-2}$. These latter authors concluded that this excess emission is produced by the interaction of MeV CR prorons from the SNR with the ambient cold gas. However, this explanation seems quite puzzling since it would require a relatively high column density of gas comparable to that of the molecular cloud in the northeastern part of the remnant $\left(N_{\mathrm{H}_{2}} \simeq\right.$ $\left.10^{22} \mathrm{~cm}^{-2}\right)$. However, the region of enhanced emission as defined in Nobukawa et al. (2018) and the TeV gamma-ray contour by HESS (respectively the area enclosed with the dashed blue line and the green contour in Fig. 2) do not overlap. It should also be noted that an earlier analysis of Suzaku data performed by Okon et al. (2018) found a region of enhanced Fe I K $\alpha$ line emission at a different position (centering around the NE source). Since the position of the enhanced emission varies for different analyses, it is difficult to investigate its origin.

Nevertheless, the Fe I K $\alpha$ line emission is quite an informative channel for the study of low-energy CRs and could also be used to put some constraints on the CR spectra. In particular, the total flux coming from the excess measured by Nobukawa et al. (2018) can be computed as $F_{\mathrm{ex}}=\Delta \vartheta\left(I_{6.4 \mathrm{keV}}^{\mathrm{en}}-I_{6.4 \mathrm{keV}}^{\mathrm{bg}}\right)$ where $\Delta \vartheta \approx 15^{2} \operatorname{arcmin}^{2}$ is the extension of the excess region. Such a flux, if produced by CR protons in the northeastern cloud, would have been detected.

The nondetection of the iron line from the northeastern cloud can be used to impose an additional constraint on the parameter $T_{\mathrm{c}}$ introduced in Sect. 4.1. The intensity of the Fe I K $\alpha$ line emission from the region of the molecular cloud can be calculated as:

$F_{6.4 \mathrm{keV}}=\frac{M_{\mathrm{cl}}}{4 \pi D_{\mathrm{s}}^{2} m_{\mathrm{avg}}} \int_{T_{\mathrm{c}}}^{\infty} \mathrm{d} T_{\mathrm{i}} \sigma_{\mathrm{K} \alpha}\left(T_{\mathrm{i}}\right) 4 \pi J_{\mathrm{i}}\left(T_{\mathrm{i}}\right)$,

where $\sigma_{\mathrm{K} \alpha}\left(T_{\mathrm{i}}\right)$ is the cross section of the $\mathrm{K} \alpha$ line emission (Tatischeff et al. 2012). A lower limit for $T_{\mathrm{c}}$ can be obtained by imposing $F_{6.4 \mathrm{keV}}<F_{\text {ex }}$. This gives $T_{\mathrm{c}}>30 \mathrm{MeV}$, which is consistent with the range of possible values of $T_{\mathrm{c}}$ reported in Table 3 .

\section{Discussion and conclusion}

Here, we derive constraints on the $\mathrm{CR}$ proton and electron spectra in the region of the SNR W28. The gamma-ray emission from the MCs in the region demonstrates that an excess of CR protons is present there. The present study focuses on the northeastern cloud, which is interacting with the SNR shock.

Vaupré et al. (2014) tentatively proposed that the excess of $\mathrm{CR}$ protons in the region might also explain the enhanced ionization rate observed from the northeastern cloud. However, CR electrons and/or X-ray photons coming from the SNR shock can also contribute to the ionization.

Here, we develop a model for the transport of X-ray photons into the cloud and demonstrate that their contribution to the observed ionization rate is negligible. Moreover, even though we cannot completely rule out CR electrons as the main ionizing agents, we show that the most natural explanation for the enhanced ionization rate is explained in terms of interactions of CR protons.

To explain both the gamma-ray emission from the cloud and the enhanced ionization rate, the spectrum of protons in the cloud must extend to particle energies smaller than those constrained by gamma-ray observations $(\gtrsim 1 \mathrm{GeV})$. However, an extrapolation of the spectrum of an order of magnitude only in particle energy would suffice to explain both high- and low-energy observations. This makes protons the most plausible dominant ionizing agents inside the cloud.

The minimal scenario that would simultaneously explain high- and low-energy data would require the presence of CR protons characterized by a single power-law spectrum of slope $\delta_{\mathrm{p}} \sim 2.8$ extending over a very broad energy range, spanning from $T_{\mathrm{c}} \lesssim 100 \mathrm{MeV}$ up to several tens of TeV: almost six orders of magnitude in energy. In fact, the presence of a spectral break cannot be ruled out below a particle energy of $\approx 1 \mathrm{GeV}$ because the ionization rate depends only on the integral of the CR spectrum over particle energy. The presence of a break would require modification of the value of $T_{\mathrm{c}}$ accordingly in order to correctly reproduce the observed ionization rate, but this would not affect the main conclusion of our study in any significant way.

The work presented in this paper shows how the combination of high- and low-energy observations of SNR/MC systems can be used as a very powerful tool to gather information on the $\mathrm{CR}$ spectrum at specific locations in the Galaxy over an energy range of unprecedented breadth. More studies in this direction are desirable, as they will shed light on the process of CR acceleration and escape from SNR shocks (Gabici \& Montmerle 2015).

Acknowledgements. The authors would like to thank C. Ceccarelli and T. Montmerle for many years of useful discussions. VHMP would like to thank M. K. Erdim, N. Tsuji, H. Yoneda, N. Cesur, and Prof. S. Safi-Harb for helpful discussions during the DIAS Summer School in High-Energy Astrophysics 2018 and D. Allard and V. Tatischeff for support during my thesis. This project has received funding from the European Union's Horizon 2020 research and innovation programme under the Marie Skłodowska-Curie Grant agreement No. 665850, from the Agence Nationale de la Recherche (Grant ANR17-CE31-0014), and from the Observatory of Paris (Action Fédératrice CTA). GM acknowledges the support received through The Grants ASI/INAF n. 201714-H.O and SKA-CTA-INAF 2016.

\section{References}

Abdo, A. A., Ackermann, M., Ajello, M., et al. 2010, ApJ, 718, 348 Aharonian, F. 2004, Very High Energy Cosmic Gamma Radiation: A Crucial Window on the Extreme Universe (World Scientific)

Aharonian, F., Akhperjanian, A. G., Bazer-Bachi, A. R., et al. 2008, A\&A, 481, 401

Borkowski, K. J., Lyerly, W. J., \& Reynolds, S. P. 2001, ApJ, 548, 820 
Brogan, C. L., Gelfand, J. D., Gaensler, B. M., Kassim, N. E., \& Lazio, T. J. W. 2006, ApJ, 639, L25

Caselli, P., Walmsley, C. M., Terzieva, R., \& Herbst, E. 1998, ApJ, 499, 234

Claussen, M. J., Frail, D. A., Goss, W. M., \& Gaume, R. A. 1997, ApJ, 489, 143

Crutcher, R. M. 1999, ApJ, 520, 706

Cui, Y., Yeung, P. K. H., Tam, P. H. T., \& Pühlhofer, G. 2018, ApJ, 860, 69

Dalgarno, A., Yan, M., \& Liu, W. 1999, ApJS, 125, 237

Dame, T. M., Hartmann, D., \& Thaddeus, P. 2001, ApJ, 547, 792

Dogiel, V. A., Chernyshov, D. O., Tatischeff, V., Cheng, K.-S., \& Terrier, R 2013, ApJ, 771, L43

Drury, L. O'C. 2017, ArXiv e-prints [arXiv:1708. 08858]

Dubner, G. M., Velázquez, P. F., Goss, W. M., \& Holdaway, M. A. 2000, AJ, 120, 1933

Dumas, G., Vaupré, S., Ceccarelli, C., et al. 2014, ApJ, 786, L24

Fujita, Y., Ohira, Y., Tanaka, S. J., \& Takahara, F. 2009, ApJ, 707, L179

Gabici, S., \& Montmerle, T. 2015, 34th International Cosmic Ray Conference (ICRC2015), 34, 29

Gabici, S., Casanova, S., Aharonian, F. A., \& Rowell, G. 2010, SF2A-2010 Proceedings of the Annual meeting of the French Society of Astronomy and Astrophysics, 313

Gabici, S., Evoli, C., Gaggero, D., et al. 2019, Int. J. Mod. Phys. D, 28, $1930022-$ 339

Hewitt, J. W., Yusef-Zadeh, F., \& Wardle, M. 2008, ApJ, 683, 189

Kafexhiu, E., Aharonian, F., Taylor, A. M., \& Vila, G. S. 2014, Phs. Rev. D, 90, 123014
Krause, J., Morlino, G., \& Gabici, S. 2015, 34th International Cosmic Ray Conference (ICRC2015), 34, 518

Kovalenko, A. V., Pynzar', A. V., \& Udal'Tsov, V. A. 1994, Astron. Rep., 38, 95

Li, H., \& Chen, Y. 2010, MNRAS, 409, L35

Maloney, P. R., Hollenbach, D. J., \& Tielens, A. G. G. M. 1996, ApJ, 466, 561

Matsunaga, K., Mizuno, N., Moriguchi, Y., et al. 2001, PASJ, 53, 1003

Morlino, G., \& Gabici, S. 2015, MNRAS, 451, L100

Morrison, R., \& McCammon, D. 1983, ApJ, 270, 119

Nava, L., \& Gabici, S. 2013, MNRAS, 429, 1643

Nobukawa, K. K., Nobukawa, M., Koyama, K., et al. 2018, ApJ, 854, 87

Ohira, Y., Murase, K., \& Yamazaki, R. 2011, MNRAS, 410, 1577

Okon, H., Uchida, H., Tanaka, T., et al. 2018, PASJ, 70, 35

Padovani, M., Galli, D., \& Glassgold, A. E. 2009, A\&A, 501, 619

Phan, V. H. M., Morlino, G., \& Gabici, S. 2018, MNRAS, 480, 5167

Recchia, S., Phan, V. H. M., Biswas, S., \& Gabici, S. 2019, MNRAS, 485, 2276

Rho, J., \& Borkowski, K. J. 2002, ApJ, 575, 201

Schuppan, F., Röken, C., \& Becker Tjus, J. 2014, A\&A, 567, A50

Tatischeff, V., Decourchelle, A., \& Maurin, G. 2012, A\&A, 546, A88

Vaupré, S., Hily-Blant, P., Ceccarelli, C., et al. 2014, A\&A, 568, A50

Velázquez, P. F., Dubner, G. M., Goss, W. M., \& Green, A. J. 2002, AJ, 124 2145

Vink, J. 2012, A\&ARv, 20, 49

Zhou, P., Safi-Harb, S., Chen, Y., et al. 2014, ApJ, 791, 87 